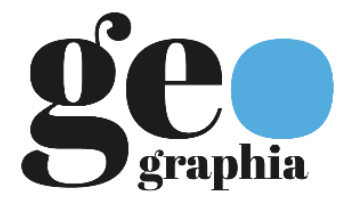

ARTIGOS

\title{
GEOQUÍMICA E PETROGRAFIA DE DEPÓSITOS QUATERNÁRIOS DA PORÇÃO SW DA PRAIA BRAVA, ARMAÇÃO DOS BÚZIOS, RJ, BRASIL: EDFRX E DIFRAÇÃO A LASER
}

\author{
Diogo Gabriel Sperandio ${ }^{1}$ \\ Universidade Federal de Minas Gerais \\ Cristiane Heredia Gomes ${ }^{2}$ \\ Universidade Federal do Pampa
}

Guilherme Pazinato Dias ${ }^{3}$

Universidade Federal do Pampa

Enviado em 19 mar. 2020 | Aceito em 19 mai. 2021

Resumo: Este artigo apresenta os dados geoquímicos e petrográficos de sedimentos praiais da região SW da Praia Brava (PB) em Armação dos Búzios-RJ. Buscamos discutir os processos de intemperismo químico e proveniência, bem como, analisar o ambiente geoquímico e a sua relação com as fontes autóctones. Neste estudo foram utilizadas análises petrográficas, parâmetros texturais na distribuição de partículas, geoquímica e estatística com auxílio de Laser Difraction e Fluorescência de Raios-X com Energia Dispersiva. Os sedimentos estudados são compostos essencialmente de granadas, biotita, piroxênio (diopsídio e augita?), epidoto, monazita, hornblenda, ilmenita, zircão, rutilo, titanita, silimanita, cianita, além de quartzo. Os sedimentos investigados são reflexo dos litotipos encontrados regionalmente, indicando possivelmente uma forte correlação entre as rochas-fonte e os depósitos sedimentares. 0 ambiente de oxirredução é demarcado pela presença de óxidos de Fe, provavelmente hematita. 0 índice de alteração calculado suporta um grau moderado, onde micas são preservadas, totalmente ou em partes.

Palavras-Chave: Sedimentos Inconsolidados; Depósitos Praiais; Intemperismo Químico.

\section{GEOCHEMISTRY AND PETROGRAPHY OF QUATERNARY DEPOSITS FROM SW OF PRAIA BRAVA BEACH, ARMAÇÃO DOS BÚZIOS, RJ, BRAZIL: EDFRX AND LASER DIFRACTION}

Abstract: This article presents the geochemical and petrographic data of beach sediments from the SW region of Praia Brava (PB) in Armação dos Búzios-RJ. We seek to discuss the processes of chemical weathering and provenance, as well as to analyze the geochemical environment and its relationship with autochthonous sources. In this study, petrographic analyzes, textural parameters in particle distribution (Laser Difraction), geochemistry (X-Ray Fluorescence with Dispersive Energy) and statistics were used. The studied sediments are essentially composed of garnets, biotite, pyroxene (diopside and augite?), epidote, monazite, hornblende, ilmenite, zircon, rutile, titanite, silimanite, kyanite, and quartz. The investigated sediments are similar to the lithotypes found regionally, thus possibly indicating a strong correlation between source rocks and sedimentary deposits. The redox environment is marked by the presence of Fe oxides, probably hematite. The alteration index supports a moderate degree, where micas are preserved, in whole or in part.

Keywords: Unconsolidated Sediments, Beach Deposits; Chemical watering.

1. Doutorando, Universidade Federal de Minas Gerais, e-mail: dsperandio@ufmg.br, Orcid: https://orcid.org/0000-00015791-7379.

2. Doutora em Ciências, ênfase em Geoquímica, Laboratório de Mineralogia e Petrografia, Universidade Federal do Pampa, e-mail: cristianegomes@unipampa.edu.br, Orcid: https://orcid.org/0000-0001-7297-913X.

3. Graduando, Universidade Federal do Pampa, e-mail: gui.pazinato.dias@gmail.com, Orcid: https://orcid.org/0000-00025074-4222. 
GEOQUÍMICA Y PETROGRAFÍA DE DEPÓSITOS CUTERNARIOS DE LA PORCIÓN SW DE PRAIA BRAVA, ARMAÇÃO DOS BÚZIOS, RJ, BRASIL: EDFRX Y DIFFRACCIÓN LÁSER

Resumen: Este artículo presenta los datos geoquímicos y petrográficos de sedimentos de playa de la región SO de Praia Brava (PB) en Armação dos Búzios-RJ. Buscamos discutir los procesos de meteorización química y procedencia, así como analizar el entorno geoquímico y su relación con fuentes autóctonas. En este estudio se utilizaron análisis petrográficos, parámetros texturales en distribución de partículas, geoquímica y estadística con la ayuda de Difracción Láser y Fluorescencia de Rayos X con Energía Dispersiva. Los sedimentos estudiados están compuestos fundamentalmente por granate, biotita, piroxeno (¿diópsido y augita?), Epidota, monacita, hornblenda, ilmenita, circón, rutilo, titanita, silimanita, cianita, además de cuarzo. Los sedimentos investigados son un reflejo de los litotipos encontrados regionalmente, lo que posiblemente indique una fuerte correlación entre las rocas generadoras y los depósitos sedimentarios. El entorno redox está delimitado por la presencia de óxidos de Fe, probablemente hematita. El índice de cambio calculado admite un grado moderado en el que se conservan las micas, ya sea total o parcialmente.

Palabras clave: Sedimentos no consolidados; Depósitos en la playa; Meteorización química.

Introdução

A composição e a maturidade de sedimentos siliciclásticos é controlada por inúmeros fatores, incluindo as rochas-fontes, os processos associados ao intemperismo, transporte e deposição deste material. Diversos trabalhos mostraram que a morfometria e geoquímica de sedimentos são ferramentas apropriadas para desvendar a fonte (CISLAGHI e BISCHETTI, 2019; MA et al., 2017) a configuração e evolução tectônica (VERMA e ARMSTRONG-ALTRIN, 2013; ROSER et al., 1996; 1986) taxas de denudação e processos erosionais das áreas fontes. A assinatura química, aliada a estudos de petrografia de sedimentos nos permite compreender os processos de interação água-rochasedimento e, por sua vez, a dinâmica do ambiente geoquímico em questão (GOMES et al., 2018b). 0 conhecimento acerca dos parâmetros geoquímicos tem sido aplicado em sedimentos para traçar o ambiente deposicional e as paleocondições das rochas fontes (CLAVERT e PEDERSEN, 1993; JONES e MANNING, 1994; GHABRIAL et al., 2012). Por sua vez, a compreensão da proveniência e transporte dos sedimentos está intimamente correlacionada aos parâmetros geoquímicos e, também, aos físicos de caracterização mineralógica e morfométrica que tangem aos processos dinâmicos exógenos (NESBITT et al., 1996; NAGARAJAN et al., 2007). A caracterização do ambiente geoquímico a partir da assinatura geoquímica de seus sedimentos tem sido fruto de diversos trabalhos (ALBA et al., 2008; DE LACERDA e MARINS, 2006; GONÇALVES et al., 2017; GOMES, et al., 2018a;2020). Esta caracterização permite uma abordagem sistemática sobre os processos geológicos atuantes da região, principalmente em termos de minerais guias.

Os sedimentos investigados neste trabalho representam os depósitos praiais da Praia Brava localizada em Armação dos Búzios, estado do Rio de Janeiro (Brasil), oriundos da ampla e vasta diversidade geológica registrada no Cabo de Búzios (HEILBRON et al.,1982; BULHÕES e FERNANDEZ, 2012). O substrato rochoso regional é caracterizado pela Sequência Supracrustal da Sucessão Búzios (Figura 1), paragnaisses Neoproterozóicos (1 Ga - $541 \mathrm{Ma}$ ) da Sucessão Búzios e Sucessão Palmital (SCHIMITT, 2012).

A Sucessão Búzios é um espesso pacote de rochas metassedimentares aluminosas, composta por cianita-silimanita-granada-biotita gnaisses, intercalações de rochas calcissilicáticas bandadas, granada anfibolitos, clinopiroxênio-granada anfibolitos e granada quartzitos (SCHIMITT, 2012). Por outro lado, a Sucessão Palmital é caracterizada por (granada)-silimanita-gnaisses com intercalações de rochas metassedimentares calcissilicáticas e camadas de quartzito. Já a Unidade Forte de São 
Mateus está disposta sob duas formas de ocorrência: i) sob pacotes espessos de até 50 metros de granada-anfibolito com níveis calcissilicáticos e ii) intercalada com a Sucessão Búzios em forma de camadas delgadas de até cinco metros. Os litotipos destas unidades ocorrem metamorfisados em fácies anfibolito até granulito e constituem sucessões com no mínimo 500 metros de espessura (HEILBRON et al.,1982). A paragênese das rochas, conforme Schmitt et al., (2003), está associada a ambientes deposicionais marinhos (600 Ma, Neoproterozóico). Entre 525 e 490 milhões de anos ocorre os eventos metamórficos descritos por Schimitt et al (2004), em condições de alta pressão e temperatura. Os eventos de metamorfismo estão associados à Orogenia Búzios (HEILBRON et al.,1982) os quais são relativamente jovens em comparação com outros eventos do brasiliano. Localmente ainda ocorrem enxames de diques basálticos em Cabo de Búzios como registro da quebra do supercontinente e abertura da porção Sul do Oceano Atlântico (TETZNER et al., 2001; DUTRA, 2006).

\section{Figura 1 - Mapa geológico da Região do Armação dos Búzios}

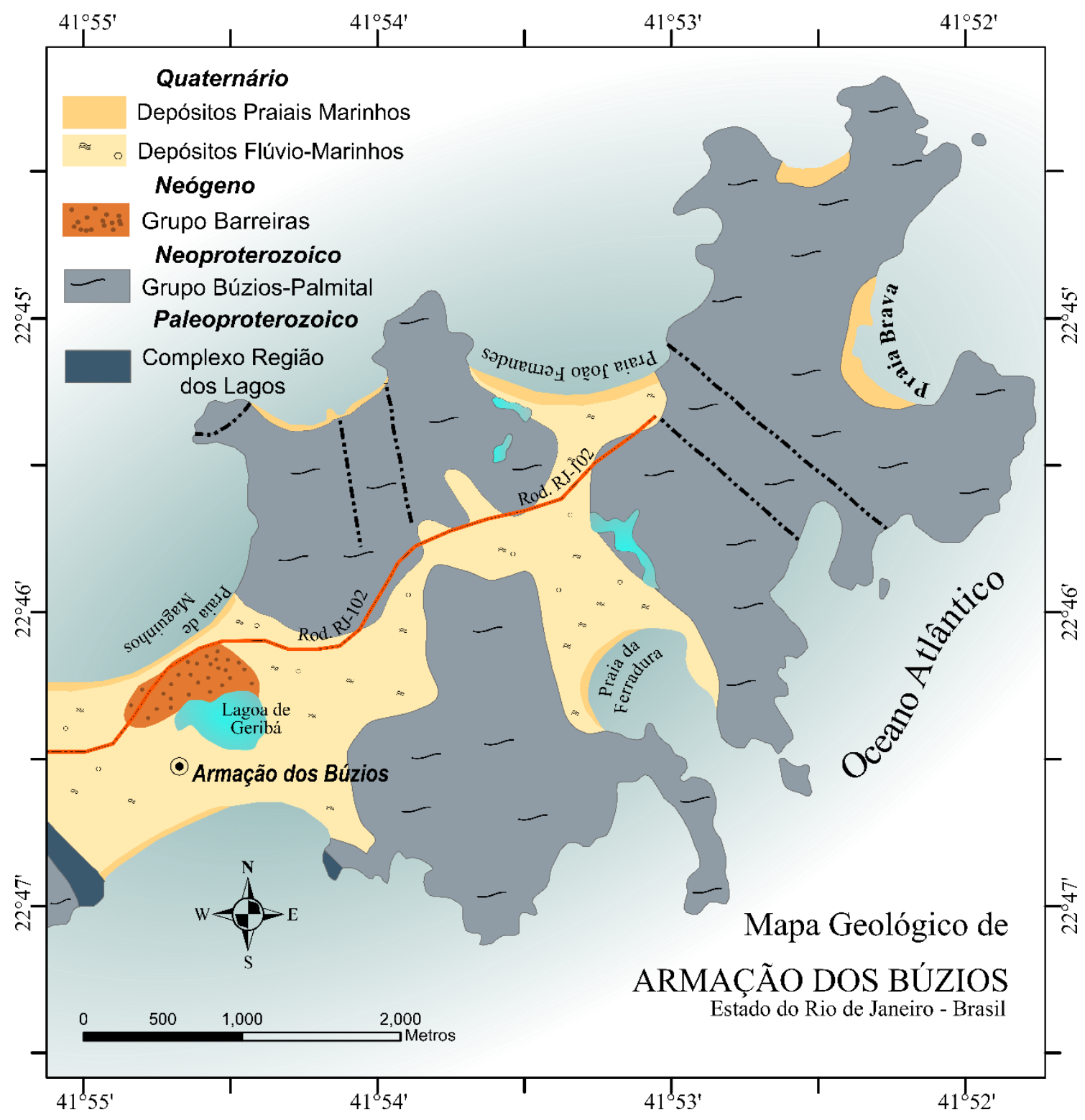


A proposta do presente estudo é identificar as características geoquímicas dos sedimentos da praia Brava, relacionando-os com as rochas fonte e as condições do ambiente quando da formação desses sedimentos. Os resultados são discutidos em termos de intemperismo e proveniência da área fonte.

\section{Área de estudo}

A Praia Brava (PB) está situada à leste da região central de Búzios entre as praias de João Fernandes e Olho de Boi, com aproximadamente $0,5 \mathrm{~km}$ de extensão. Em termos morfológicos e morfodinâmicos, Bulhões e Fernandez. (2016) caracterizam a Praia Brava como um ambiente que, apesar de ser intermediário de baixa energia, é instável, sujeito a eventos episódicos de tempestade, e por vezes, apresentando aspecto erodido permanente. Por outro lado, o ambiente costeiro é caracterizado por frequentes alterações espacial e temporalmente, seja em sua expressão geomorfológica ou geológicas (DAVIDSON-ARNOTT et al., 2019; CHRISTOFOLETTI, 1980; STEERS, 1971). Ainda, para o mesmo autor, a diversidade morfológica litorânea deve-se, também, as ações erosivas e deposicionais.

A área de estudo na Praia Brava compreende a porção face da praia (ÓRTEGA-SANCHEZ et al., 2008; MASSELINK e PULEO, 2006), caracterizada por depósitos de sedimentos quaternários (Figura 1; MORAIS, 2001; SANTIAGO, GUERRA e VILELA, 2011) que estão sob influência direta de processos dinâmicos costeiros, tais como ondas e marés. Na PB ocorre predomínio de areias finas, de coloração avermelhada, moderadamente a bem selecionadas nas extremidades da praia, enquanto que na porção central ocorre predomínio de areias finas a médias bem selecionadas.

Figura 2 - Imagem de Satélite com a localização dos pontos amostrados na praia Brava

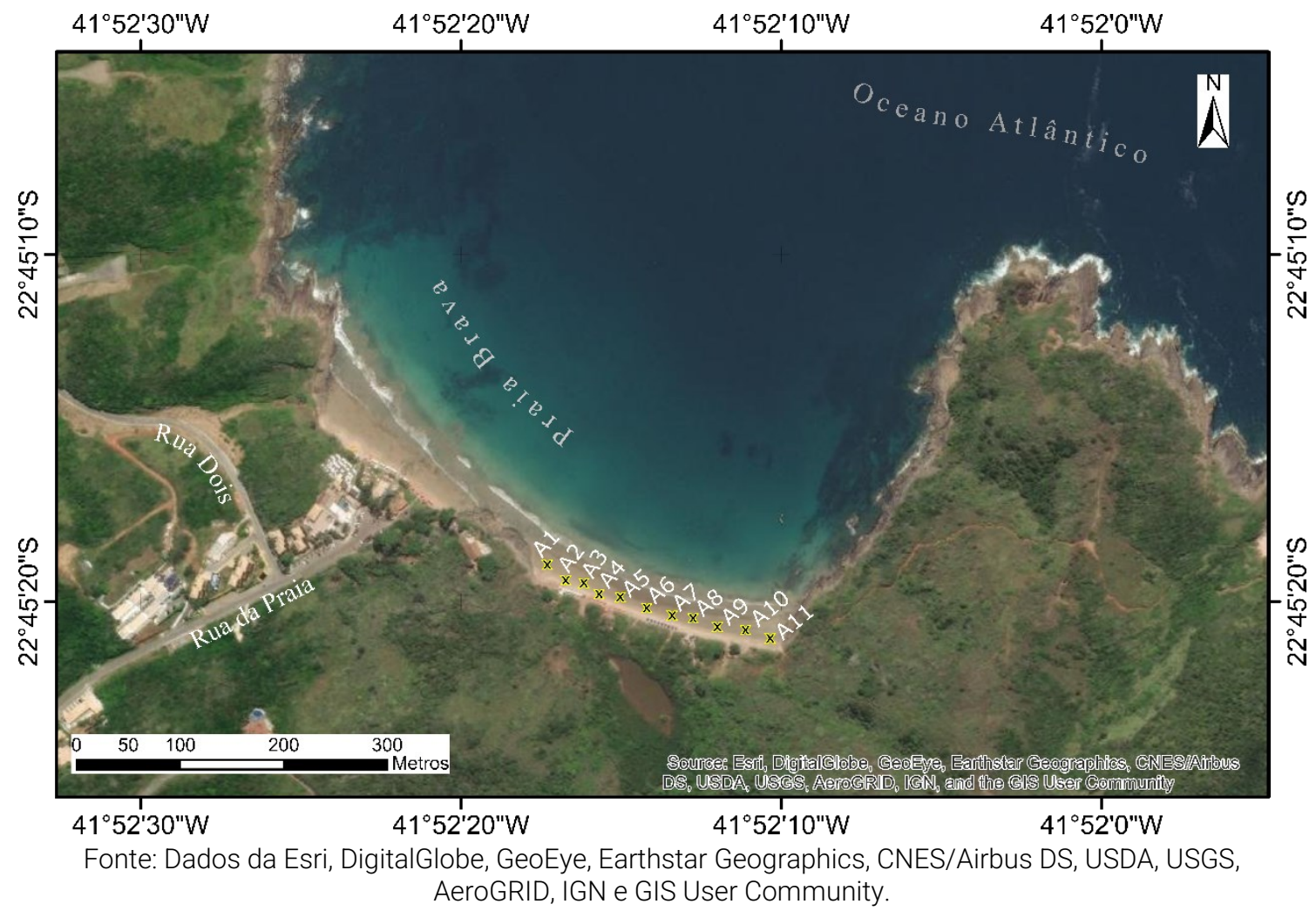




\section{Materiais e métodos}

Um total de onze amostras de sedimentos foram investigados ao longo da zona praial na porção sudeste da Praia Brava-RJ (Figura 2). Foram coletadas aproximadamente $200 \mathrm{~g}$ de sedimentos em cada amostragem realizada, com uma profundidade média de $10 \mathrm{~cm}$ e espaçamento de 20 metros entre cada ponto de coleta. Todas as amostras foram preservadas e deixadas para secar em temperatura ambiente por três dias em formas de polipropileno de acordo com os critérios da Embrapa (2006).

As análises petrográficas foram realizadas no Laboratório de Mineralogia e Petrografia da Universidade Federal do Pampa (UNIPAMPA) com o auxílio de lupa binocular e microscópio óptico.

As análises de distribuição de partículas e de geoquímica foram realizadas no Laboratório de Lavra e Tratamento de Minérios (LATRAM/UNIPAMPA). A distribuição de partículas foi mensurada com o auxílio de um Analisador de Partículas a Laser (Microtrac S3500; ZHANG et al., 2019; LI et al., 2013;), em uma amplitude de 0,01 a 10.000 um com 3mW de potência nominal e tempo de medição entre de 30 segundos. Adicionou-se alíquotas de $1 \mathrm{~g}$ de sedimento no porta amostra e, após a passagem da amostra pelos feixes de laser do aparelho, esse forneceu os dados de tamanho de partícula por intensidade. A partir do método foram caracterizadas propriedades morfométricas dos sedimentos, tais como: classes granulométricas, esfericidade e arredondamento.

Para as análises geoquímicas, cada uma das amostras foi quarteada a fim de se obter porções de 50 g. destinados à análise de Fluorescência de Raios-X com Energia Dispersiva (EDFRX). O EDFRX, (Bruker S1 Turbo SD; NYAMBURA et al., 2013; FLOOD et al., 2017; GOMES et al., 2020; OUJIDI et al., 2021), foi utilizado por um tempo de 120 segundos em uma velocidade de 100.000 contagens por segundo para cada uma das amostras. 0 equipamento utiliza um ânodo de Ag, que permite a medição de 25 elementos e um detector Silicon Deriva Detector (SDD) de $10 \mathrm{~mm}^{2}$ com refrigeração termoelétrica e resolução de 145 eV a MnKa (BAZAES CASANOVA, 2013). Foi realizado triplicatas para cada uma das onze amostras, conforme recomendado pela literatura, afim de se garantir uma excepcional representatividade dos resultados sendo os dados apresentados neste trabalho uma média das triplicatas de cada amostra tendo sido excluídos os elementos em estavam em concentração elementar menor do que o limite de detecção do aparelho.

O intemperismo normalmente relacionado a sedimentos pode ser estimado a partir da quantidade de álcalis presentes na amostra (NESBITT et al., 1996). Uma estimativa do grau de intemperismo em sedimentos é sugerida pelo cálculo do Índice de Alteração - IA $\left.\left(\mathrm{Al}_{2} \mathrm{O}_{3} /\left(\mathrm{Al}_{2} \mathrm{O}_{3}+\mathrm{CaO}+\mathrm{Na}_{2} \mathrm{O}+\mathrm{K}_{2} \mathrm{O}\right)\right) \times 100\right)$. Rochas e minerais pouco ou desprovidos de intemperismo apresentam IA próximo de 50, valores de IA inferiores a 60 são associados a amostras com baixo graus de intemperismo e para aquelas amostras com IA entre 60 e 80 o intemperismo é moderado (TAYLOR e MCLENNAN, 1985).

O método de Análise de Componentes Principais (PCA) foi usado como técnica estatística multivariada. Este método é comumente utilizado para investigar a variabilidade dos dados (ex. CHENG et al., 2006; SCHEIB et al., 2011). A análise de correlação e de componentes principais foi realizada com o Statistical Package Statsoft (versão 10). PCA é um método de redução variável que produz um pequeno número de variáveis artificiais, conhecidos como Componentes Principais (PCs). Cada PC representa um determinado número de variáveis artificiais dos dados, e os primeiros dois PCs usualmente respondem pela maior variação no grupamento de dados analisados (REIMAN et al., 2008). 


\section{Resultados e discussão}

\section{Composição mineralógica dos sedimentos}

Fotomicrografias dos minerais presentes nas amostras estudadas são apresentadas na figura 3. A caracterização mineralógica das amostras revelou que os sedimentos são compostos, em sua maioria, por granadas, biotita, piroxênios (diopsídio e augita), epidoto, monazita, hornblenda, ilmenita, zircão, rutilo, titanita, silimanita, cianita, óxido de ferro, além de quartzo.

A augita (figura 3a) $\left(\mathrm{Ca}_{x} \mathrm{Mg}_{y} \mathrm{Fe}_{z}\right)\left(\mathrm{Mg}_{y 1} \mathrm{Fe}_{z 1}\right) \mathrm{Si}_{2} \mathrm{O}_{6} ;$ onde $0.4 \leq x \leq 0.9 ; x+y+z=1$ e y1 $+\mathrm{z} 1=1$ ) (fórmula a partir de MORIMOTO et al., 1981), clinopiroxênio monoclínico (POMEROL et al., 2013), é subarredondada com moderada esfericidade e brilho resinoso (figura 3a), por vezes, é vítreo. 0 epidoto (figura 3b) $\left\{\mathrm{Ca}_{2}\right\}\left\{\mathrm{Al}_{2} \mathrm{Fe}^{3+}\right\}\left(\mathrm{Si}_{2} \mathrm{O}_{7}\right)\left(\mathrm{SiO}_{4}\right) \mathrm{O}(\mathrm{OH})$ tem coloração esverdeada, brilho graxo e é subarredondado. O zircão (figura 3c) ( $\mathrm{ZrSiO}_{4}$ ), ortosilicato tetragonal (DEER, HOWIE e ZUSSMAN, 1986), é incolor, arredondado e levemente alongado, lembrando prismas tetragonais. Tal como vasta literatura relata, (FEDO et al., 2003; NASDALA et al., 1996; GARNAR, 1994; CARROL, 1953) o zircão mostra-se relativamente resistente aos processos exógenos, e portanto, alterações significativas na estrutura e forma (dissolução, por exemplo) são dificuldadas. A muscovita (figura 3d) $\left(\mathrm{KAl}_{2}\left(\mathrm{Si}_{3} \mathrm{Al}\right) \mathrm{O}_{10}(\mathrm{OH}, \mathrm{F})_{2}\right)$, filossilicato monoclínico (POMEROL et al., 2013), apresenta brilho nacarado, baixa esfericidade e arredondamento irrelevante.

As granadas $\left(\mathrm{X}_{3} \mathrm{Z}_{2}\left(\mathrm{SiO}_{4}\right)_{3}\right.$; onde $\mathrm{X}=\mathrm{Mg}, \mathrm{Ca}, \mathrm{Fe}(\mathrm{II}), \mathrm{Mn}(\mathrm{II})$ e $\mathrm{Z}=\mathrm{Al}, \mathrm{Fe}(\mathrm{III}), \mathrm{Cr}(\mathrm{III}), \mathrm{V}(\mathrm{III}), \mathrm{GEIGER}, 2013$; GALOISY, 2013; GREW et al., 2013) (figuras 3e e 3f) apresentam-se subangulares de moderada a baixa esferidade. Apresenta microinclusões máficas, sugerindo uma série de processos cristalogenéticos e cristaloquímicos durante os eventos metamórficos que atuaram regionalmente.

Cianita e silimanita ( $\mathrm{Al}_{2} \mathrm{SiO}_{5}$ ) (CLARK, ROBERTSON e BIRCH, 1957; RAO et al., 2001; WINKLER et al., 2001) (Figura 3j e 3k) polimorfos de alumínio (POMEROL et al., 2013), são facilmente encontradas nos sedimentos investigados. A cianita ocorre como mineral alongado de brilho vítreo e clivagem marcante - muitas vezes evidenciada por degraus no mineral. A silimanita apresenta-se subaredondada com coloração amarela-marrom. O leucoxênio (Figura 3i), é subesférico e medianamente arredondado. Aqui interpretamos o leucoxênio como produto da alteração e mistura de óxidos de Fe-Ti. Provavelmente um produto da alteração da ilmenita, rutilo (Figura 3h) ou mesmo do esfeno (a partir de MÜCKE e BHADRA CHAUDHURI, 1991; MORAD e ALDAHAN, 1986; LYND, 1960). Embora a IMA (International Mineralogical Association) tenha descreditado o leucoxênio como um mineral (IMA 06-C; BURKE, 2006; GREY, WATTS, e BAYLISS, 1994) neste trabalho optamos pela designação com o objetivo de diferenciá-lo de outros minerais, especialmente o rutilo.

É quase que imperativa a comparação entre a composição mineralógica dos sedimentos investigados e a composição mineralógica do arcabouço geológico adjacente. Ocorre uma concordância substancial entre a composição mineralógica dos sedimentos e das rochas adjacentes. Conforme Guerra et al., (2005) discutem, toda a região situada entre o Cabo Frio e o Cabo de Búzios, nas condições atuais do nível do mar, não apresentam condições ideais para que o aporte sedimentológico fluvial direto seja robusto. Ao contrário, a compartimentação atual dos sistemas praiais regionais acaba favorecendo trocas laterais de sedimentos (SCHMITT et al., 2012; GUERRA et al., 2005). 
Figura 3 - Fotomicrografias dos minerais descritos nas amostras coletadas na Praia Brava vistas em aumento de 10X em luz natural. a) augita subaredondada de moderada esfericidade; b) grão de epidoto de coloração esverdeada; c) zircão incolor arredondado e levemente alongado, lembrando prismas tetragonais; d) muscovita com brilho nacarado; e) granada com inclusões de máficos; f) granada anédrica com inclusões máficas; g) biotita com brilho vítreo; h) rutilo com típico brilho adamantino e coloração vermelho-escuro; i) leucoxenio, provavelmente produto da alteração da Ilmenita, rutilo ou esfeno; j) silimanita subarredondada; k) cristal alongado de cianita com clivagem marcante.
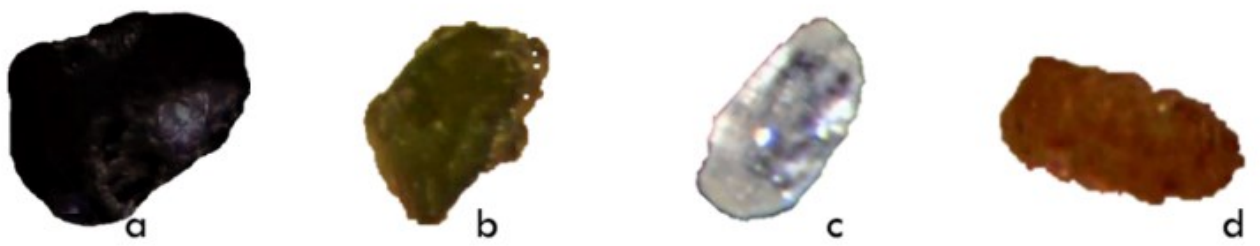
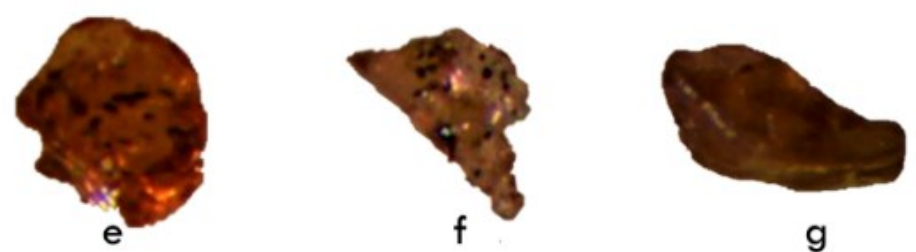

g
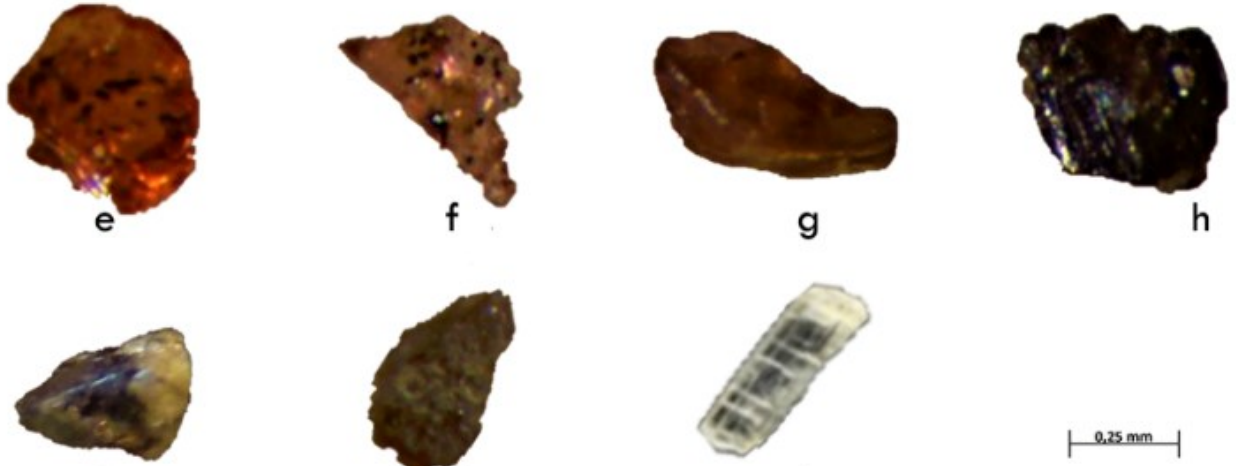

i



h

d

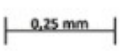

\section{Análise de Partículas}

As propriedades morfométricas dos sedimentos caracterizadas com o auxílio do Analisador de Partículas à Laser, lupa eletrônica e microscópio petrográfico, indicam tamanho de grão compreendido na fração areia fina à média-fina com distribuições, em geral, simétricas mesocúrticas. A esfericidade média das amostras é moderada a baixa. O arredondamento dos minerais foi classificado como subangular (Figura 4).

Os aspectos morfométricos, tais como: tamanho, arredondamento e esfericidade estão diretamente relacionados com as propriedades físicas de cada um dos minerais e sua resposta à processos exógenos, como o intemperismo e são largamente utilizados para a caracterização destes ambientes (NESBITT et al., 1996; GOMES et al., 2018a). Por outro lado, o arranjo atômico de cada um dos minerais, também, reage de modo distinto a estes processos. Deste modo, existe uma íntima relação entre as propriedades físico-químicas da mineralogia dos sedimentos com o ambiente geoquímico e os processos exógenos atuantes (WRONKIEWICZ e CONDIE, 1987; ALLOWAY et al., 1990).

A DF mede a distribuição do tamanho das partículas por meio da variação angular da luz difundida a partir da interação de um laser com as partículas amostrais (MALVERN, 2011). Neste sentido, partículas maiores dispersam a luz em ângulos pequenos em relação ao feixe laser, por outro lado, partículas menores produzem grandes ângulos de dispersão. Assim, estes dados são utilizados para o cálculo do tamanho das partículas e o seu padrão de dispersão, com base na teoria de difusão da luz de Mie (MALVERN, 2011). As análises obtidas junto ao analisador de partículas à laser dos sedimentos investigados mostram uma distribuição simétrica mesocúrtica concentrada no tamanho 
areia fina (figura 4) compatível com um grau de intemperismo baixo a médio atuante (NESBITT et al., 1996; GOMES et al., 2018a).

Figura 4 - Análise granulométrica das amostras investigadas obtidas a partir do Analisador de Partículas a Laser (Laser Difraction - LD).



\section{Espectrometria por Fluorescência de Raios-X com Energia Dispersiva (EDFRX)}

As análises geoquímicas realizadas por EDFRX para as onze amostras investigadas são apresentadas na Tabela 1. Em termos de elementos maiores, os valores de ferro obtidos variaram de $30,4(\mathrm{~A} 1)$ a 13,7\% (A11), com uma média geral de 19,57\% e sendo até $46 \%$ mais elevada do que a quantidade no cianita-silimanita-granada-gnaisse. Isto sugere um reflexo dos constituintes ferrosilicáticos e enriquecimento de óxido de ferro nesses sedimentos, corroborando assim com a petrografia. Aliado a isto, no âmbito dos minerais ferromagnesianos, o magnésio apresenta valores entre 1,64 e 4,09\% com exceção das amostras A1 e A4 (7,05 e 6,96\%, respectivamente). Os valores médios de Al são 7,40\% tendo seu pico em 9,41\% (amostra A11). A variação dos valores de alumínio nos sedimentos investigados foi atribuída a presença de polimorfos de alumínio constituintes tais como cianita e silimanita e, também ao epidoto. As amostras A11, A8 e A10 apresentam os valores mais elevados de Ca (13,8; 10,9; e 10,4, respectivamente), com média geral de $8,13 \%$, sendo associado aos piroxênios (diopsídio e augita). Os valores de potássio obtidos variam de zero a 0,04\% e foram atribuídos a dissolução em maior ou menor grau de muscovita. Os valores de Mn são próximos a $1.1 \%$ para todas as amostras, com exceção da amostra A1 $(2,23)$. Os valores médios de titânio nos sedimentos investigados estão próximos a 1,5\%. 0 titânio possivelmente está associado a minerais metamórficos como a ilmenita (óxido de ferro e titânio). As concentrações de silício variam de 16,5 (A2) a 20,9\% (A9), sendo associadas a diversificação da proveniência do aporte sedimentar (Sucessão Búzios e Palmital).

Tabela 1 - Resultado das análises geoquímicas para as amostras de sedimentos investigadas por EDFRX e do cianitasilimanita-granada-gnaisse (CSGG) para comparação

\begin{tabular}{ccccccccccccc}
\hline $\begin{array}{c}\text { Amostra/ } \\
\text { elementos } \\
\text { (\%) }\end{array}$ & A1 & A2 & A3 & A4 & A5 & A6 & A7 & A8 & A9 & A10 & A11 & CSGG \\
\hline SiO2 & 17,0 & 16,5 & 18,9 & 17,6 & 16,9 & 17,1 & 18,2 & 18,3 & 20,9 & 18,7 & 22,4 & 10,4 \\
Al2O3 & 7,50 & 7,53 & 6,53 & 7,86 & 5,64 & 6,42 & 7,48 & 7,07 & 8,06 & 7,92 & 9,41 & 3,52 \\
Fe203 & 30,4 & 20,7 & 19,8 & 21,4 & 18,9 & 18,9 & 17,4 & 16,1 & 18,8 & 19,2 & 13,7 & 13,4 \\
MnO & 2,23 & 1,29 & 1,11 & 1,11 & 0,99 & 1,14 & 1,07 & 0,85 & 1,07 & 1,14 & 0,80 & 0,17 \\
MgO & 7,05 & 2,73 & 1,64 & 6,96 & 0,00 & 2,53 & 3,85 & 0,00 & 2,83 & 0,00 & 4,09 & 3,6 \\
CaO & 4,65 & 4,52 & 6,25 & 5,25 & 5,51 & 9,50 & 9,51 & 10,9 & 9,09 & 10,4 & 13,8 & 3,98 \\
TiO2 & 2,83 & 1,42 & 1,38 & 2,04 & 1,78 & 2,05 & 1,18 & 1,00 & 1,16 & 1,19 & 0,51 & 2,97 \\
P2O5 & 0,00 & 0,09 & 0,00 & 0,00 & 0,00 & 0,02 & 0,00 & 0,04 & 0,00 & 0,00 & 0,00 & 0,24 \\
K20 & 0,00 & 0,00 & 0,00 & 0,00 & 0,00 & 0,00 & 0,00 & 0,03 & 0,00 & 0,00 & 0,04 & 3,08 \\
Cl & 0,16 & 0,18 & 0,15 & 0,10 & 0,08 & 0,16 & 0,12 & 0,16 & 0,11 & 0,09 & 0,12 & 0,14 \\
Cr & 0,02 & 0,02 & 0,00 & 0,00 & 0,00 & 0,02 & 0,01 & 0,02 & 0,00 & 0,00 & 0,02 & 0 \\
Co & 0,00 & 0,95 & 0,93 & 1,05 & 0,80 & 0,90 & 0,83 & 0,74 & 0,91 & 0,95 & 0,68 & 0,59 \\
Ce & 0,00 & 0,28 & 0,13 & 0,00 & 0,00 & 0,13 & 0,11 & 0,56 & 0,08 & 0,26 & 0,15 & 0,23 \\
Cd & 0,00 & 0,00 & 0,00 & 0,00 & 0,08 & 0,00 & 0,00 & 0,10 & 0,00 & 0,11 & 0,07 & 0,14 \\
Mo & 0,00 & 0,02 & 0,02 & 0,01 & 0,03 & 0,00 & 0,03 & 0,01 & 0,00 & 0,00 & 0,00 & 0,03 \\
\hline IA & 61,73 & 62,49 & 51,10 & 59,95 & 50,58 & 40,33 & 44,03 & 39,34 & 47,00 & 43,23 & 40,54 & 33,27 \\
\hline
\end{tabular}

Em relação à concentração dos elementos menores observados, o cobalto apresenta concentração uniforme nas amostras, em geral, muito próximo à 1\%, exceto para a amostra A1 (zero\%). O cromo quando detectado apresenta concentrações variando entre 0,01 e 0,02\% nas amostras $A 1, A 2, A 6, A 7, A 8$ e $A 11$. 0 cério tem distribuição heterogênea não regular nas amostras, não sendo detectado nas amostras $A 1, A 4$ e $A 5$, enquanto que nas demais amostras variou de 0,08 a $0,56 \%$. 
Os valores de IA calculados para os sedimentos investigados (Tabela 1) variam de 40,33 (A6) a 62,49 (A2), indicando que esses sedimentos não estão alterados apresentam um baixo ou moderado desgaste químico, principalmente nas amostras A1 e A2. Neste sentido, o IA reflete o grau de resistência dos silicatos na formação de argilominerais (FEDO et al., 1995).

\section{Análise estatística bivariada}

Os dados geoquímicos obtidos por EDFRX foram analisados em termos de correlação estatística descritiva para uma melhor compreensão da correlação positiva ou negativa entre os elementos dos sedimentos investigados (Tabela 2). Sillanpää (1972) e Alloway et al. (1990) sugerem que o conteúdo total e suas correlações no material de origem refletem seu conteúdo nas amostras de sedimentos. A correlação estatística no presente trabalho foi baseada em correlações bivariadas e foi expressa em coeficiente de correlação de Pearson (p). O coeficiente de correlação de Pearson mede o grau de correlação e sua direção, se positiva ou negativa, entre duas variáveis de escala métrica. Esta correlação apresenta-se pela variável $p$ assumindo valores entre -1 e 1 , conforme Equação 1:

$$
\rho=\frac{\sum_{i=1}^{n}\left(x_{i}-\bar{x}\right)\left(y_{i}-\bar{y}\right)}{\sqrt{\sum_{i=1}^{n}\left(x_{i}-\bar{x}\right)^{2}} \cdot \sqrt{\sum_{i=1}^{n}\left(y_{i}-\bar{y}\right)^{2}}}=\frac{\operatorname{cov}(X, Y)}{\sqrt{\operatorname{var}(X) \cdot \operatorname{var}(Y)}}
$$

Sendo que:

$$
\bar{x}=\frac{1}{n} \cdot \sum_{i=1}^{n} x_{i} \quad \bar{y}=\frac{1}{n} \cdot \sum_{\substack{\text { e, também, } \\ i=1}}^{n} y_{i}{ }_{\text {são médias aritméticas das variáveis } \mathrm{X} \text { e } \mathrm{Y} \text {. }}
$$

Assim, a análise de correlação indica a relação entre duas vaiáveis e os valores sempre serão entre +1 e -1. Por outro lado, o sinal indica a direção, se a correlação é positiva ou negativa, e o tamanho da variável indica a força da correlação.

A interpretação em relação ao seu tamanho frente a força de correlação é disposta da seguinte maneira: $p \geq 0.9$ positivo ou negativo indica uma correlação muito forte; $0.7 \leq p \geq 0.9$ positivo ou negativo indica uma correlação forte; $0.5 \leq p \geq 0.7$ positivo ou negativo indica uma correlação moderada; $0.3 \leq p \geq 0.5$ positivo ou negativo indica uma correlação fraca; $0 \leq p \geq 0.3$ positivo ou negativo indica uma correlação desprezível (SILLANPÄÄ, 1972; ALLOWAY et al., 1990). 
Tabela 2 - Correlação estatística descritiva de Pearson para os sedimentos investigados

\begin{tabular}{|c|c|c|c|c|c|c|c|c|c|c|c|c|c|c|c|c|c|c|}
\hline & MgO & Al203 & $\mathrm{SiO2}$ & P2O5 & $\mathbf{S}$ & Cl & $\mathrm{CaO}$ & TiO2 & $\mathrm{Cr}$ & MnO & $\mathrm{Fe} 2 \mathrm{O3}$ & Co & $Y$ & $\mathbf{Z r}$ & Nb & Mo & Cd & $\mathrm{Ce}$ \\
\hline MgO & 1 & & & & & & & & & & & & & & & & & \\
\hline $\mathrm{Al} 2 \mathrm{O3}$ & 0.37 & 1 & & & & & & & & & & & & & & & & \\
\hline $\mathrm{SiO} 2$ & -0.05 & 0.70 & 1 & & & & & & & & & & & & & & & \\
\hline P2O5 & -0.20 & -0.08 & -0.39 & 1 & & & & & & & & & & & & & & \\
\hline $\mathbf{S}$ & 0.08 & 0.58 & 0.68 & -0.08 & 1 & & & & & & & & & & & & & \\
\hline Cl & 0.12 & -0.08 & -0.28 & 0.66 & 0.02 & 1 & & & & & & & & & & & & \\
\hline $\mathrm{CaO}$ & -0.32 & 0.50 & 0.73 & -0.21 & 0.71 & -0.16 & 1 & & & & & & & & & & & \\
\hline TiO2 & 0.53 & -0.44 & -0.71 & -0.09 & -0.52 & 0.18 & -0.73 & 1 & & & & & & & & & & \\
\hline $\mathrm{Cr}$ & 0.23 & 0.19 & -0.09 & 0.53 & 0.47 & 0.75 & 0.22 & 0.04 & 1 & & & & & & & & & \\
\hline MnO & 0.57 & -0.06 & -0.45 & -0.02 & -0.38 & 0.35 & -0.58 & 0.80 & 0.23 & 1 & & & & & & & & \\
\hline $\mathrm{Fe} 2 \mathrm{O3}$ & 0.83 & 0.28 & -0.15 & -0.17 & 0.04 & 0.21 & -0.39 & 0.63 & 0.30 & 0.84 & 1 & & & & & & & \\
\hline Co & -0.49 & -0.08 & 0.08 & 0.17 & -0.12 & -0.29 & 0.12 & -0.47 & -0.48 & -0.75 & -0.80 & 1 & & & & & & \\
\hline $\mathbf{Y}$ & 0.68 & 0.14 & -0.15 & -0.32 & -0.28 & 0.03 & -0.22 & 0.42 & 0.08 & 0.61 & 0.59 & -0.56 & 1 & & & & & \\
\hline $\mathbf{Z r}$ & 0.33 & -0.24 & -0.31 & -0.15 & -0.08 & 0.10 & -0.34 & 0.58 & 0.27 & 0.67 & 0.67 & -0.89 & 0.40 & 1 & & & & \\
\hline Nb & 0.68 & 0.43 & 0.31 & -0.33 & 0.36 & 0.00 & 0.10 & 0.32 & 0.19 & 0.27 & 0.49 & -0.29 & 0.22 & 0.12 & 1 & & & \\
\hline Mo & -0.19 & -0.53 & -0.40 & 0.16 & -0.36 & -0.10 & -0.40 & -0.07 & -0.25 & -0.22 & -0.30 & 0.26 & 0.09 & -0.05 & -0.70 & 1 & & \\
\hline Cd & -0.60 & 0.05 & 0.21 & -0.07 & 0.32 & -0.39 & 0.50 & -0.45 & -0.04 & -0.42 & -0.32 & 0.03 & -0.47 & 0.13 & -0.39 & -0.09 & 1 & \\
\hline $\mathrm{Ce}$ & -0.58 & 0.06 & 0.05 & 0.58 & 0.14 & 0.41 & 0.43 & -0.52 & 0.39 & -0.37 & -0.44 & 0.17 & -0.42 & -0.20 & -0.48 & -0.07 & 0.51 & 1 \\
\hline Th & -0.36 & -0.10 & 0.38 & -0.08 & 0.51 & 0.03 & 0.32 & -0.47 & 0.06 & -0.47 & -0.23 & -0.02 & -0.48 & 0.11 & -0.28 & 0.19 & 0.48 & 0.34 \\
\hline $\mathbf{R h}$ & -0.67 & -0.11 & 0.29 & 0.15 & 0.30 & -0.23 & 0.50 & -0.73 & -0.16 & -0.97 & -0.90 & 0.78 & -0.68 & -0.66 & -0.38 & 0.30 & 0.39 & 0.42 \\
\hline
\end{tabular}

A correlação estatística descritiva apresentada na Tabela 2 indica uma correlação positiva muito forte entre o Mn-Fe (0,96), Fe-Ti (0,88); Al-Si $(0,70)$; Mn-Ti $(0,80)$; Ca-Si $(0,73)$; S-Ca $(0,71)$; $\mathrm{Cl}-\mathrm{Cr}$ $(0,75)$ e Co-Rh $(0,78)$. As correlações positivas moderadas foram observadas para Fe-Mg $(0,54) ; \mathrm{Y}-\mathrm{Fe}$ (0,54); Ti-Zr (0,59); Ca-Cd (0,50); Ce-Cd (0,51); P-Ce (0,58); Th-S (0,51); Mn-Mg (0,55); Al-S (0,59); Cl-P $(0,67)$.

A correlação estatística revela, também, forte correlação negativa entre Rh-Mn (-0.97); Fe-Rh (0.93); Co-Zr; Ti-Si; Ca-Ti; Co-Mg; Fe-Ca. A correlação moderadamente negativa se dá nos seguintes elementos: Cd-Mg; Zr-Rh; Rh-Mg; Fe-Co; Y-Rh; Y-Zr; Mo-Nb; Mo-Al; Fe-Si; Ti-S; Fe-S; Mn-Ca; Ce-Ti; Y-Co; Ce-Mg (Tabela 2).

As principais trocas iônicas estão correlacionadas com estes coeficientes de correlação $(p)$ medidos e dentro de ordens iguais ou semelhantes. Neste caso, tal como Gomes et al. (2018b) indica, sugere-se que aumentos ou diminuições, espontâneas e simultâneas, dos vetores catiônicos seja dado em função das trocas iônicas da própria assembleia mineral constituinte dos sedimentos aqui estudados.

Para a análise química das propriedades dos sedimentos investigados, foi aplicada a Análise de Componentes Principais (PCA), com base na matriz de correlação entre os componentes e as variáveis padronizadas (Sarma, Durlofsky e Aziz, 2008; Gazley et al., 2015; Yang e Cheng, 2015). Portanto, nos sedimentos da praia Brava, nove (09) PCs representam 100\% da variação dos resultados obtidos (Tabela 3 ). Os três primeiros PCs apresentam autovalores maiores que 1 , 
representando 87,38\% da variação (Tabela 3; Figura 5). O primeiro e o segundo componentes principais (PC1 e PC2) dos sedimentos investigados são o resultado da combinação linear de 19 variáveis estudadas, e os dois PCs explicaram 44,49\% e 25,94\% da variância, respectivamente (Tabela 3).

Tabela 3 - Matriz de correlação de autovalores e relação estatística dos sedimentos da praia Brava, RJ, investigados

\begin{tabular}{ccccc}
\hline PCs & Autovalores & Variância total \% & Autovalores acumulado & Acumulado \% \\
\hline 1 & 4.00 & 44.49 & 4.00 & 44.49 \\
2 & 2.33 & 25.94 & 6.33 & 70.43 \\
3 & 1.52 & 16.94 & 7.86 & 87.38 \\
4 & 0.48 & 5.38 & 8.34 & 92.76 \\
5 & 0.28 & 3.18 & 8.63 & 95.95 \\
6 & 0.21 & 2.43 & 8.85 & 98.39 \\
7 & 0.07 & 0.79 & 8.92 & 99.18 \\
8 & 0.04 & 0.53 & 8.97 & 99.71 \\
9 & 0.02 & 0.28 & 9.00 & 100.00 \\
\hline
\end{tabular}

A PCA também produz vetores próprios, conhecidos como coeficientes ou cargas dos componentes principais (Figura 5). Eles descrevem a importância relativa de um componente, por exemplo, um elemento químico e sua variabilidade entre um grupo de dados. Os autovetores calculam automaticamente a pontuação de cada PC. Os valores das cargas do elemento determinam a pontuação de um ponto de amostragem. Assim, o agrupamento de elementos de alta carga fornece altas pontuações e o agrupamento de elementos de baixa carga útil fornece pontuações baixas. 0 número de vetores próprios calculados será igual ao número de variáveis utilizadas, que neste estudo é o número de elementos e parâmetros químicos analisados (REIMANN et al., 2008).

Para a PC1 (variação de 44,50\%) dos elementos e parâmetros dos sedimentos da praia Brava, pontuações altas são proporcionais a elementos de alta carga $(>0,2)$, por exemplo, $\mathrm{Al}, \mathrm{Si}, \mathrm{Ca}, \mathrm{Cd}, \mathrm{Ce}$, Co, Th e Rh (Figura 4). As pontuações mais baixas são equivalentes às taxas mais baixas $(<0,19)$, como $\mathrm{Cl}, \mathrm{P}$ e Mo. As pontuações mais altas para PC2 estão relacionadas a fortes cargas positivas (> $0,2)$ para Fe, Mg e Mn, com baixas taxas relacionadas a Cl e P (Figura 5). 
Figura 5 - Projeção das varáveis nas Componentes Principais (PC1 e PC2) dos autovetores nos sedimentos investigados

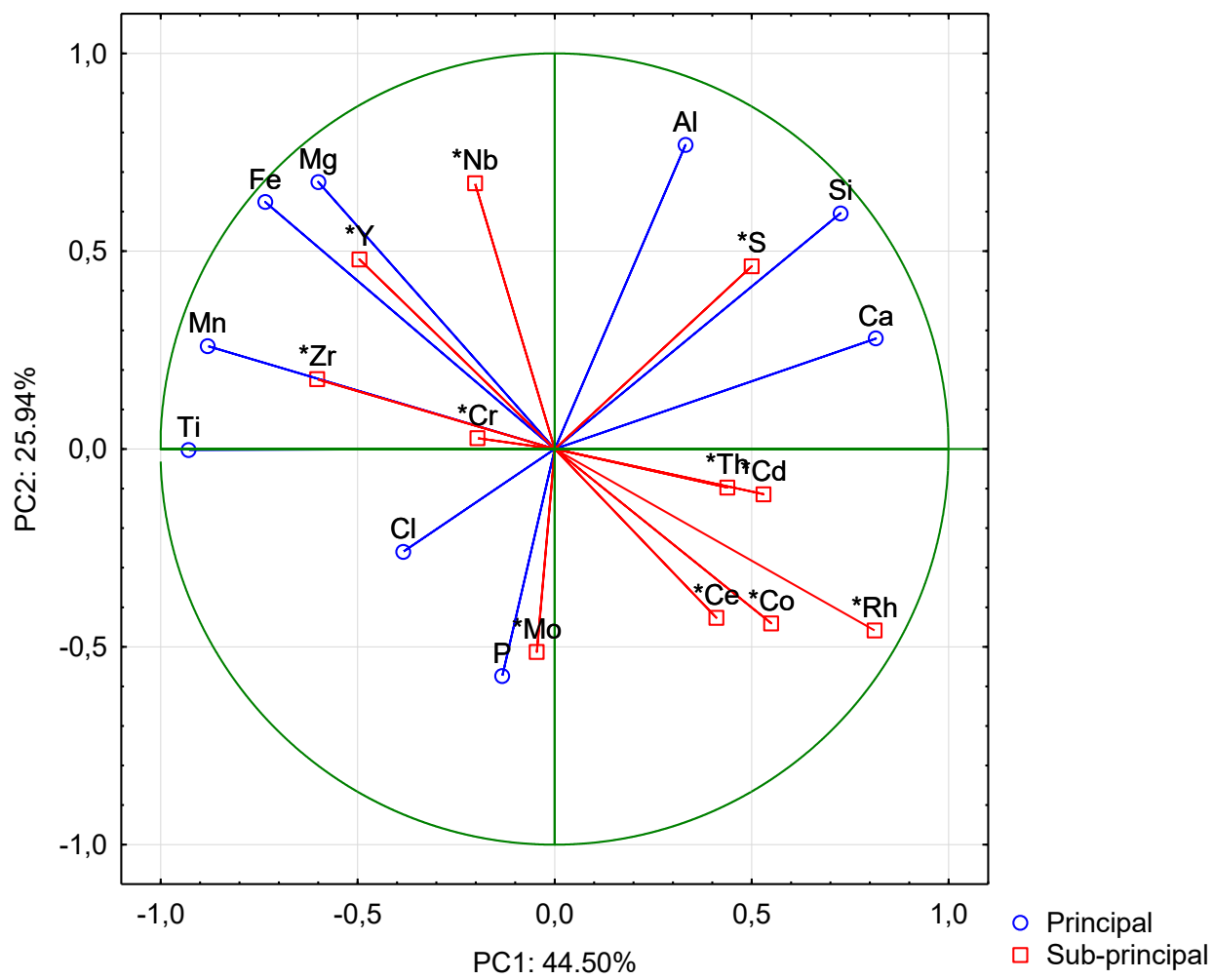

\section{Conclusão}

Analisamos características morfométricas como tamanho de grão, composição mineral, forma dos grãos e geoquímica dos sedimentos praiais da porção SW da Praia Brava em Armação dos Búzios. A mineralogia das amostras estudadas correlaciona-se intrinsecamente com o arcabouço geológico regional. Os dados sedimentométricos obtidos revelam uma distribuição simétrica mesocúrtica com esfericidade média e arredondamento subangular para as amostras. Isto sugere um grau atuante de intemperismo baixo a médio para as amostras investigadas. Dados de IA calculados revelam índices baixos a moderados para o desgaste químicos das amostras, refletindo o grau de resistência dos silicatos na gênese de argilominerais.

Os dados apresentados e discutidos ao longo do manuscrito nos levam a crer que a principal fonte de sedimentos para a praia Brava seja proveniente de processos erosivos do arcabouço rochoso adjacente. Por outro lado, em maior ou menor grau, a plataforma continental pode igualmente ter um importante papel no aporte de sedimentos, embora não esteja claro qual é o tamanho desta influência.

\section{Agradecimentos}

Agradecemos o apoio financeiro da Fundação de Amparo à Pesquisa do Estado do Rio Grande do Sul (PROBITI-FAPERGS), Programa de Desenvolvimento Acadêmico (PDA-UNIPAMPA) e Conselho Nacional de Ciência e Tecnologia (PIBIT-CNPq). 


\section{Referências}

ALBA, J. M. F.; SOUZA FILHO, C. R.; FIGUEIREDO, B. R. (2008) Análise da assinatura geoquímica de solos e de sedimentos de corrente no Vale do Ribeira (SP) por meio de um sistema de informação geográfica. Revista Brasileira de Geociências, v. 38, n. 1, p. 66-77.

ALLOWAY, B. J. (1990) Heavy metals in soils: trace metals and metalloids in soils and their bioavailability. Springer Science \& Business Media, v. 27, n. 7, p. 120-135.

BAZAES CASANOVA A. A. W. (2013) Comparación de corrección teórica de [...] de rayos x en minerales y suelos. [thesis]. Santiago, Chile. Facultad de Ciencias quimicas y farmacia/Universidad de Chile, 200p.

BULHOES, E. M. R.; FERNANDEZ, G. B. (2016) Aspectos Morfodinâmicos Em Praias De Enseada: Estudo De Caso Em Armação Dos Búzios, Brasil. Revista Brasileira de Geomorfologia, v. 17, n. 2, p. 253271.

BURKE, E.A.J. (2006) A mass discreditation of GQN minerals. The Canadian Mineralogist. v. 44, n. 6, p. 1557-1560

CALVERT, S. E.; PEDERSEN, T. F. (1993) Geochemistry of Recent Oxic and Anoxic Sediments: Implications for the Geological Record. Marine Geology, v. 113, p. 67-88.

CARROLL, D. (1953) Weatherability of zircon. Journal of Sedimentary Research, v. 23, n. 2, p. 106-116. CHENG, Q.; JING, L.; PANAHI, A. (2006) Principal component analysis with optimum order sample correlation coefficient for image enhancement. International Journal of Remote Sensing, v. 27, n. 16, p. 3387-3401.

CHRISTOFOLETTI, A. (1980) Geomorfologia. São Paulo: Blucher, 188 p.

CISLAGHI, A. e BISCHETTI, G. B. (2019) Source areas, connectivity, and delivery rate of sediments in mountainous-forested hillslopes: A probabilistic approach. Science of the Total Environment, v. 652, p. 1168-1186.

CLARK, S. P.; ROBERTSON, E. C. e BIRCH, F. (1957) Experimental determination of kyanite-sillimanite equilibrium relations at high temperatures and pressure, American Journal of Science, v. 255, p. 628640.

DAVIDSON-ARNOTT, R.; BAUER, B. e HOUSER, C. (2019) Introduction to coastal processes and geomorphology. Cambridge university press, $460 \mathrm{p}$.

DE LACERDA, L. D e MARINS, R. V. (2006) Geoquímica de sedimentos e o monitoramento de metais na plataforma continental nordeste oriental do Brasil. Geochimica Brasiliensis, v. 20, n. 1, p. 120-132.

DEER, W.A., HOWIE, R.A., ZUSSMAN, J. (1986) Introduction to the Rock-Forming Minerals, Mineralogical Society. $498 \mathrm{p}$.

DUTRA T. (2006) Petrogênese dos basaltos de baixo-TiO2 do Enxame de Diques da Serra do Mar na Região dos Lagos, RJ. [dissertação]. Rio de Janeiro, Instituto de Geociências/UFRJ, 111 p.

EMBRAPA - Empresa Brasileira de Pesquisa Agropecuária. Centro Nacional de Pesquisa de Solos. (2006) Manual de métodos de análise de solo. v. 1, 36 p.

FEDO, C. M.; SIRCOMBE, K. N. e RAINBIRD, R. H. (2003). Detrital zircon analysis of the sedimentar record. In: J. M. HANCHAR, P. W. O. HOSKIN (Eds.), Zircon: Reviews in Mineralogy and Geochemistry, v. 53, p. 277-303.

FEDO, C. M.; NESBITT, H. W. e YOUNG, G. M. (1995) Um ravelling the effects of potassium metasomatism in sedimentary rocks and paleosols, with implications for paleoweathering conditions and provenance. Geology, v. 23, p. 921-924.

FLOOD, R. P.; BARR, I. D.; WELTJE, G. J.; ROBERSON, S.; RUSSELL, M. I.; MENEELY, J. D. \& ORFORD, J. D. (2017). Late Holocene depositional variability and provenance in the lower Ganges-Brahmaputra delta. Sedimentary Geology, v. 155, p. 317-342.

GALOISY, L. (2013) Garnet: From stone to star. Elements, v. 9, p. 453-456.

GARNAR, T. E. (1994) Zirconium and hafnium minerals. In: CARR D. D. (org.). Industrial Minerals and Rocks. Indiana: Society for mining, metallurgy, and exploration, p. 1159-1165.

GAZLEY, M. F.; COLLINS, K. S.; ROBERSTON, J.; HINES, B. R.; FISHER, L. A., e MCFARLANE, A. (2015). Application of principal component analysis and cluster analysis to mineral exploration and mine 
geology. In AusIMM New Zealand Branch Annual Conference (Vol. 2015, pp. 131-139). Dunedin New Zealand.

GEIGER, C.A. (2013) Garnet: A key phase in nature, the laboratory, and technology. Elements, v. 9, p. 447-452.

GHABRIAL, D. S.; SAMUEL, M. D. e MOUSSA, H. E. (2013) Geochemistry and tectonic setting of early Pan-African metamorphosed volcano-sedimentary sequence in southern Solaf zone, SW Sinai, Egypt. Arabian Journal of Geosciences, v. 6, n. 10, p. 3635-3649.

GOMES, C.H.; SPERANDIO, D.G.; DIAS, G.P. e VIÇOZZI, A.P. (2020) Soil geochemistry of the municipality necropolis of Mata, RS, by fluorescence of x-rays by dispersive energy. Ciência e Natura, v. 42, n. e11, p. 1- 21.

GOMES, C. H.; ALMEIDA, D. P. M. e SPERANDIO, D. G. (2018a) Geoquímica de Sedimentos da Confluência das Bacias Hidrográficas Baixo Jacuí e Vacacaí-Mirim, Caçapava do Sul-RS: Implicações para Proveniência e Intemperismo Químico. Anuário do Instituto de Geociências, v. 41, n.3, p. 470-482. GOMES, C. H.; SPERANDIO, D. G.; DESSART, R. L. e GIUSTI, D. D. (2018b) Detection and Evaluation of metals in soil under influence of mining by Dispersive Energy X-ray Fluorescence Spectrometry (EDXRF), Lavras do Sul/RS. Ciência e Natura, v. 40, n. e70, p. 1-10.

GONÇALVES, M. A.; TABORDA, R. e BATISTA, M. J. (2017) Geoquímica de sedimentos de corrente na prospecção de mineralizações de W-Sn e Metais preciosos na Zona Centro Ibérica. In: Anais do X Congresso Ibérico de Geoquímica - Lisboa. Disponível em: http://xcig.ineg.ptl. Acessado em: 15 mar. 2020.

GREY, I. E.; WATTS, J. A. e BAYLISS, P. (1994). Mineralogical nomenclature: pseudorutile revalidated and neotype given. Mineralogical Magazine, v. 58, n. 393, p. 597-600.

GREW, E.S.; LOCOCK, A.J.; MILLS, S.J.; GALUSKINA, I.O.; GALUSKIN, E.V. e HÅLENIUS, U. (2013) Nomenclature of the garnet supergroup. American Mineralogist, v. 98, p. 785-811.

HEILBRON, M. P. C. L; CHRISPIM, S. I.; ALVES, R. P. e SIMOES. L. S. A. (1982) Geology of Cape Buzios State of Rio de Janeiro. Anais da Academia Brasileira de Ciências, v. 54, n. 3, p. 553-562.

JONES, B.; MANNING, D. A. C. (1994) Comparison of Geochemical Indices Used for the Interpretation of Palaeoredox Conditions in Ancient Mudstones. Chemical Geology, v. 111, p. 111-129.

LI, X.; WANG, Y.; LI, B.; FENG, C.; CHEN, Y.; SHEN, Z. (2013). Distribution and speciation of heavy metals in surface sediments from the Yangtze estuary and coastal areas. Environmental earth sciences, v. 69, n. 5, p. 1537-1547.

LYND, L.E. (1960) Alteration of ilmenite. Economic Geology, v. 55, n. 5, p. 1064-1068.

MA, K.; HU, S.; WANG, T.; ZHANG, B. e QIN, S. (2017) Sedimentary environments and mechanisms of organic matter enrichment in the Mesoproterozoic Hongshuizhuang Formation of northern China. Palaeogeography Palaeoclimatology Palaeoecology, v. 475, p. 176-187.

MALVERN PANALYTICAL LDT. (2011) Difração a Laser - Laser Difraction LD. Disponível em: https://www.malvernpanalytical.com/br/products/technology/light-scattering/laser-

diffraction.Acessado em: 22 jun. 2019.

MASSELINK, G. e PULEO, J. A. (2006) Swash zone morphodynamics. Cont. Shelf Res. v. 26, p. 661680

MORAD, S. e ALDAHAN, A. A. (1986) Alteration of detrital Fe-Ti oxides in sedimentary rocks. GSA Bulletin, v. 97, n. 5, p. 567-578.

MORAIS, R. M. O., (2001). Estudo faciológico da Formação Barreiras na região entre Maricá e Barra de Itabapoana, estado do Rio de Janeiro. Dissertação (Mestrado) - Programa de Pós-graduação em Geologia, Universidade Federal do Rio de Janeiro, Rio de Janeiro. 113 p.

MORIMOTO, N.; FABRIES, J.; FERGUSON, A. K.; GINZBURG, I. V.; ROSS, M.; SEIFERT, F. A.; ZUSSMAN, J.; AOKI, K. e GOTTARDI, G. (1988) Nomenclature of Pyroxenes. American Mineralogist, v. 73, p. 11231133

MÜCKE, A. e BHADRA CHAUDHURI, J.N. (1991) The continuous alteration of ilmenite through pseudorutile to leucoxene. Ore geology reviews, v. 6, n. 1, p. 25-44.

NAGARAJAN, R.; ARMSTRONG-ALTRIN, J. S.; NAGENDRA, R.; MADHAVARAJU, J. e MOUTTE, J. (2007) Petrography and geochemistry of terrigenous sedimentary rocks in the Neoproterozoic 
Rabanpalli Formation, Bhima Basin, Southern India: implications for paleoweathering conditions, provenance and source Rock composition. Journal-Geological Society of India, v. 70, n. 2, p. 297-312. NASDALA, L.; PIDGEON, R. T. e WOLF, D. (1996) Heterogeneous metamictization of zircon on a microscale. Geochimica et Cosmochimica Acta, v. 60, n. 6, p. 1091-1097.

NESBITT, H. W.; YOUNG, G. M.; MCLENNAN, S. M. e KEAYS, R. R. (1996) Effects of chemical weathering and sorting on the petrogenesis of siliciclastic sediments, with implications for provenance studies. The Journal of Geology, v. 104, n. 5, p. 525-542.

NYAMBURA, M.; TOWETT, E. K.; NYANDIKA, H.; CHACHA, R.; SHEPHERD, K. D. e GATARI, M. J. (2013). Applicability of hand-held $\mathrm{X}$-ray fluorescence analyser for rapid characterisation of soil elemental compositions. In Joint Proceedings of the 27th Soil Science Society of East Africa and the 6th African Soil Science Society Conference. Nakuru, Kenya. 20-25 de outubro 2013. p. 464-471.

ORTEGA-SÁNCHEZ, M.; FACHIN, S.; SANCHO, F. e LOSADA, M. A. (2008) Relation between beachface morphology and wave climate at Trafalgar beach (Cádiz, Spain). Geomorphology, v. 99, n. 1-4, p. 171185.

OUJIDI, B.; EL BOUCH, M.; TAHRI, M.; LAYACHI, M.; BOUTOUMIT, S.; BOUCHNAN, R.; ... e SNOUSSI, M. (2021) Seasonal and Spatial Patterns of Ecotoxicological Indices of Trace Elements in Superficial Sediments of the Marchica Lagoon following Restoration Actions during the Last Decade. Diversity, v. 13, n. 2, e. 51.

RAO, M. N.; CHAPLOT, S. L.; CHOUDHURY, N.; RAO, K. R.; AZUAH, R. T.; MONTFROOJI, W. T.; e BENNINGTON, S. M. (1999) Lattice dynamics and inelastic neutron scattering from sillimanite and kyanite Al2SiO5, Physical Review, v. B60, p. 12061.

REIMANN, C.; FILZMOSER, P.; GARRET, R. G. e DUTTER. R. (2008) Statistical Data Analysis Explained: Applied Environmental Statistics with R. [S.I.]: Wiley-Blackwell. 60 p.

ROSER, B.P.; COOPER, R.A.; NATHAN, S. e TULLOCH, A.J., (1996) Reconnaissance sandstone geochemistry, provenance and tectonic setting of the lower Paleozoic terranes of the West Coast and Nelson, New Zealand. New Zealand Journal of Geology and Geophysics, v. 879 n. 39, p. 1-16.

ROSER, B.P. e KORSCH, R.J. (1986) Determination of tectonic setting of sandstone-mudstone 872 suites using SiO2 content and K2O/Na2O ratio. Geology, v. 94, p. 635-650

SANTIAGO, N.L.; GUERRA, J.V.; VILELA, C.G. (2011) Análise sedimentológica de praias arenosas situadas entre a Ponta do Farol (Cabo Frio, RJ) e a Ponta da Emergência (Búzios, RJ). In: Anais do XIII Congresso da Associação Brasileira de Estudos do Quarternário - ABEQUA \& III Encontro do Quaternário Sulamericano. Armação dos Búzios, Rio de Janeiro. v. 1, n. 1, 9-14 de outubro de 2011.

SANTOLIN, C. V. A. (2015) Quantificação e avaliação ambiental da contaminação por metais e arsênio em sedimentos da bacia do Rio Doce-MG. [thesis]. Departamento de Química/UFMG: Belo Horizonte. $162 \mathrm{p}$.

SARMA, P.; DURLOFSKY, L. J. e AZIZ, K. (2008). Kernel principal component analysis for efficient, differentiable parameterization of multipoint geostatistics. Mathematical Geosciences, v.40, n. 1, p. 332.

SCHEIB, A. J.; LEE, J. R.; BREWARD, N. e RIDING, J. B. (2011) Reconstructing flow paths of the middle Pleistocene British ice sheet in central-eastern England: The application of regional soil geochemical data. Proceedings of the Geologists Association, v. 3, p. 432-444.

SCHIMITT, R. S. (2012) Geologia e recursos minerais das folhas Rio das Ostras e Cabo Frio SF. 24-YAIV e SF. 23-ZB-VI. CPRM: estado do Rio de Janeiro, 10 p.

SILLANPÄ̈̈, M. (1972) Distribution of trace elements in peat profiles. In: Proceedings 4th International. Peat Congress. v. 2, p. 222-228.

STEERS, J. A. (1971). Applied coastal geomorphology. London, Springer, 231 p.

POMEROL, C.; LAGABRIELLE, Y.; RENARD, M. e GUILLOT, S. (2013) Princípios de geologia: técnicas, modelos e teorias. Porto Alegre: Bookman, $256 \mathrm{p}$.

TAYLOR, S. R. e MCLENNAN, S. M. (1985) The continental crust: Its evolution and composition. London: Blackwell. $345 \mathrm{p}$.

TETZNER, W.; CAMILLO, E. J. R. e ALMEIDA, J. C. H. (2001) Tectônica Mesozóica e emplacement dos diques básicos do Cabo de Búzios, RJ. In: Anais do7º Simpósio de Geologia do Sudeste, p. 91-92. 
VERMA, S.P. e ARMSTRONG-ALTRIN, J.S. (2013) New multi-dimensional diagrams for tectonic discrimination of siliciclastic sediments and their application to Precambrian basins. Chemical Geology, v. 355, p. 117-133

WINKLER, B.; HYTHA, M.; WARREN, M.C.; MILMAN, V.; GALE, J.D. e SCHREUER, J. (2001) Calculation of the elastic constants of the Al2SiO5 polymorphs andalusite, sillimanite, and kyanite. Zeitschrift für Kristallographie, v. 216, p. 67-70.

WRONKIEWICZ, D. J. e CONDIE, K. C. (1987) Geochemistry of Archean shales from the Witwatersrand Supergroup, South Africa: source-area weathering and provenance. Geochimica et Cosmochimica Acta, v. 51, n. 9, p. 2401-2416.

YANG, J e CHENG, Q. (2015) A comparative study of independent component analysis with principal component analysis in geological objects identification, Part I: Simulations. Journal of Geochemical Exploration, v. 149, p. 127-135.

ZHANG, G.; CHENG, W.; CHEN, L.; ZHANG, H.; GONG, W. (2019). Transport of riverine sediment from different outlets in the Pearl River Estuary during the wet season. Marine Geology, v. 415, e. 105957. 